\title{
ON RICCI-HARMONIC METRICS
}

\author{
Lin Feng Wang \\ Nantong University, School of Science \\ Nantong 226007, P. R. China; wlf711178@ntu.edu.cn
}

\begin{abstract}
In this paper we study gradient Ricci-harmonic soliton metrics and quasi Ricciharmonic metrics (both metrics are called Ricci-harmonic metrics). We establish several formulas for these two metrics. Then we can show that any compact expanding or steady gradient Ricciharmonic soliton metrics are trivial in the sense that $f$ is a constant function, now the metric is harmonic Einstein. Rigid properties for the compact quasi Ricci-harmonic metric will also be proved. We derive the lower bound estimates of the scalar curvature for these two metrics in the noncompact case. Based on which we get the estimates of the growth of the potential function and the bottom of the $L_{f}^{2}$-spectrum. Eventually, we discuss the diameter estimate on the compact case.
\end{abstract}

\section{Introduction}

Let $\left(M^{m}, \mathrm{~g}\right)$ and $\left(N^{n}, \mathrm{~h}\right)$ be smooth Riemannian manifolds without boundary. We assume that $N$ is isometrically embedded into some Euclidean space $\left(N^{n}, \mathrm{~h}\right) \hookrightarrow$ $\mathbf{R}^{d}$ for $d$ large enough [29]. As in [23, 24], we identify maps $\phi: M \rightarrow N$ with $e_{N} \circ \phi: M \rightarrow \mathbf{R}^{d}$ if $e_{N}: N \rightarrow \mathbf{R}^{d}$ denotes above embedding. Hence $\phi$ can be rewritten as $\phi=\left(\phi^{\lambda}\right)_{1 \leq \lambda \leq d}$. It is well known that harmonic maps $\phi: M \rightarrow N$ are critical points for the energy functional [10]

$$
E(\phi)=\int_{M}|\nabla \phi|^{2} \mathrm{~d} V_{\mathrm{g}}
$$

where by using the Einstein convention, in a local coordinate system, $|\nabla \phi|^{2}=$ $g^{i j} \nabla_{i} \phi^{\lambda} \nabla_{j} \phi^{\lambda}$ denotes the local energy density.

In [23, 24], Müller introduced the following Ricci-harmonic flow

$$
\left\{\begin{array}{l}
\partial_{t} \mathrm{~g}(x, t)=-2 \operatorname{Ric}_{\mathrm{g}}(x, t)+2 \alpha(t) \nabla \phi(x, t) \otimes \nabla \phi(x, t), \\
\partial_{t} \phi(x, t)=\tau_{\mathrm{g}} \phi(x, t),
\end{array}\right.
$$

where $\alpha(t)>0$ depends only on $m$ and $t, \phi=\phi(t):(M, \mathrm{~g}(t)) \rightarrow(N, \mathrm{~h})$ is a family of smooth maps between $(M, \mathrm{~g}(t))$ and a fixed Riemannian manifold $(N, \mathrm{~h})$, and $\tau_{\mathrm{g}} \phi=$ trace $\nabla \mathrm{d} \phi[10]$ denotes the tension field given by the evolving metric $\mathrm{g}(t)$. When $(N, \mathrm{~h})=\left(\mathbf{R}, \mathrm{d} r^{2}\right)$, the flow (1.1) becomes the Bernhard List's flow

$$
\left\{\begin{array}{l}
\partial_{t} \mathrm{~g}(x, t)=-2 \operatorname{Ric}_{\mathrm{g}}(x, t)+2 \alpha(t) \nabla \phi(x, t) \otimes \nabla \phi(x, t), \\
\partial_{t} \phi(x, t)=\triangle_{\mathrm{g}} \phi(x, t),
\end{array}\right.
$$

doi:10.5186/aasfm.2016.4127

2010 Mathematics Subject Classification: Primary 53C21.

Key words: Gradient Ricci-harmonic soliton metric, quasi Ricci-harmonic metric, harmonic Einstein metric, scalar curvature, rigid property, volume growth, the bottom of the $L_{f}^{2}-$ spectrum, weak maximum principle at infinity, potential function.

The author was supported in part by the NSF of China (11171254), the NSF of Jiangsu Province (BK20141235). 
which is connected to the general relativity $[18,19]$. When $\phi$ is a constant function, the Bernhard List's flow becomes the well-known Ricci flow

$$
\partial_{t} \mathrm{~g}(x, t)=-2 \operatorname{Ric}_{\mathrm{g}}(x, t),
$$

which plays an important role in the proof of the Poincaré conjecture [4, 22].

For some given potential function $f$, the shifting Laplacian is $\triangle_{f}=\Delta-\nabla f \cdot \nabla$. For $\tau>0$, the $\tau$-Bakry-Émery curvature is defined by

$$
\operatorname{Ric}_{f, \tau}=\operatorname{Ric}+\operatorname{Hess} f-\frac{1}{\tau} \mathrm{d} f \otimes \mathrm{d} f .
$$

Then the ( $\infty$-)Bakry-Émery curvature is $\operatorname{Ric}_{f}=\operatorname{Ric}+\operatorname{Hess} f$. It is well known that the Bakry-Émery curvature connects with $\triangle_{f}$ by the following weighted Bochner's formula [16, 35]

$$
\begin{aligned}
\frac{1}{2} \triangle_{f}|\nabla u|^{2} & =|\operatorname{Hess} u|^{2}+\nabla \triangle_{f} u \cdot \nabla u+\operatorname{Ric}_{f}(\nabla u, \nabla u) \\
& =|\operatorname{Hess} u|^{2}+\nabla \triangle_{f} u \cdot \nabla u+\operatorname{Ric}_{f, \tau}(\nabla u, \nabla u)+\frac{1}{\tau}|\nabla u \cdot \nabla f|^{2} .
\end{aligned}
$$

Recall that a complete Riemannian metric $g$ on a smooth manifold $M$ is called a gradient Ricci soliton metric if there is a potential function $f$ and a soliton constant $\lambda$ so that $\operatorname{Ric}_{f}=\lambda \mathrm{g}$. A gradient Ricci soliton metric is called shrinking, steady or expanding, if $\lambda>0, \lambda=0$ or $\lambda<0$, respectively. Gradient shrinking Ricci soliton metrics arise often as singularity models of type $I$ singularities of the Ricci flow $[12,21,28]$. In fact, people often do not distinguish between the gradient shrinking Ricci soliton metric and the self-similar solution of the Ricci flow [9]. In fact, $\mathrm{Gu}-\mathrm{Zhu}$ [14] had shown that the complete gradient shrinking Ricci soltion metric must be the self-similar solution of the Ricci flow, so they are the same.

Many fundamental theorems in the Ricci flow have been extended to the Ricciharmonic flow. For example, no breather theorems, non-collapsing theorems [23, 24], Perelman's entropy formulas [17], monotone volume formulas [25], and volume growth estimates [44]. Müller [23] introduced the following gradient Ricci-harmonic soliton metric, which is similar to the gradient Ricci soliton metric.

Definition 1.1. Let $\left(N^{n}, \mathrm{~h}\right)$ be a fixed Riemannian manifold. A metric $\mathrm{g}$ of $M$ is a gradient Ricci-harmonic (with respect to $\mathrm{h}$ ) soliton metric, if for some map $\phi:(M, \mathrm{~g}) \rightarrow(N, \mathrm{~h})$, some potential function $f: M \rightarrow \mathbf{R}$ and some constant $\lambda, \mathrm{g}$ satisfies the following coupled system

$$
\left\{\begin{array}{l}
\operatorname{Ric}_{f}-\alpha \nabla \phi \otimes \nabla \phi=\lambda \mathrm{g}, \\
\tau_{\mathrm{g}} \phi=\nabla \phi \cdot \nabla f .
\end{array}\right.
$$

We call a gradient Ricci-harmonic soliton metric shrinking, steady or expanding, if $\lambda>0, \lambda=0$ or $\lambda<0$, respectively.

If $(N, \mathrm{~h})=\left(\mathbf{R}, \mathrm{d} r^{2}\right)$ and $\phi: M \rightarrow \mathbf{R}$ is a constant function, then the gradient Ricci-harmonic soliton metric defined in (1.3) is a gradient Ricci soliton metric. The works on the gradient Ricci soliton metric can be referred to $[2,3,11,26,27,30]$ and the references therein. When the potential function $f$ is a constant function, the gradient Ricci-harmonic soliton metric is called harmonic Einstein, which satisfies the following coupled system

$$
\left\{\begin{array}{l}
\text { Ric }-\alpha \nabla \phi \otimes \nabla \phi=\lambda \mathrm{g} \\
\tau_{\mathrm{g}} \phi=0
\end{array}\right.
$$


Obviously, harmonic Einstein metrics are natural generalizations of Einstein metrics.

Recall that a complete Riemannian metric g on a smooth manifold $M$ is called a $\tau$-quasi Einstein metric for some constant $\tau>0$ if there is a potential function $f$ and a constant $\lambda$ so that

$$
\operatorname{Ric}_{f, \tau}=\lambda \mathrm{g} .
$$

For any positive integer $\tau, \tau$-quasi Einstein metrics are closely relative to the existence of warped product Einstein manifolds [1]. In fact, let $(M, \mathrm{~g})$ and $\left(\bar{M}^{\tau}, \mathrm{h}\right)$ be two Riemannian manifolds, then for some potential function $f$ on $M$, the warped product manifold $(M \times \bar{M}, \tilde{\mathrm{g}})$ with product metric

$$
\tilde{\mathrm{g}}=\mathrm{g} \oplus \exp \left(-\frac{2 f}{\tau}\right) \mathrm{h}
$$

is Einstein if and only if $\left(\bar{M}^{\tau}, \mathrm{h}\right)$ is Einstein and the Ricci curvature tensor of $M$ satisfies the $\tau$-quasi Einstein equation (1.5) for some constant $\lambda$. The works on the quasi Einstein metric can be referred to $[5,6,7,15,36,37,38,39,40]$ and the references therein. Naturally, we will study the $\tau$-quasi Ricci-harmonic metric, which is defined as follows.

Definition 1.2. Let $\left(N^{n}, \mathrm{~h}\right)$ be a fixed Riemannian manifold. We call a metric $\mathrm{g}$ of $M \tau(\tau>0)$-quasi Ricci-harmonic (with respect to $\mathrm{h}$ ), if for some map $\phi:(M, \mathrm{~g}) \rightarrow$ $(N, \mathrm{~h})$, some potential function $f: M \rightarrow \mathbf{R}$ and some constant $\lambda$, g satisfies the following coupled system

$$
\left\{\begin{array}{l}
\operatorname{Ric}_{f, \tau}-\alpha \nabla \phi \otimes \nabla \phi=\lambda \mathrm{g}, \\
\tau_{\mathrm{g}} \phi=\nabla \phi \cdot \nabla f .
\end{array}\right.
$$

Remark 1.3. For convenience, the gradient Ricci-harmonic soliton metric and $\tau$-quasi Ricci-harmonic metric are both called Ricci-harmonic metric.

In this paper we will study the properties of Ricci-harmonic metrics. In Section 2 we establish several basic formulas. Based on these formulas, we derive rigid properties on compact or complete noncompact manifolds by using the maximum principle or the weak maximum principle at infinity, we do this in Section 3. In Section 4 we get the lower bound estimates of the scalar curvature on noncompact manifolds, these estimates play important roles in the study of geometric properties of the Ricci-harmonic metric. In the next section, we get the growth estimates of the potential function of the noncompact Ricci-harmonic metric. In the last section we study the estimates of the diameter and the bottom of the $L_{f^{-}}^{2}$-spectrum.

\section{Basic formulas}

In this section we establish basic formulas for the Ricci-harmonic metric. We firstly consider $\tau$-quasi Ricci-harmonic metrics.

Theorem 2.1. Let $\mathrm{g}$ be a $\tau$-quasi Ricci-harmonic metric defined in Definition 1.2. Then one can get

$$
\begin{aligned}
\frac{1}{2} \triangle R_{\phi}= & \frac{\tau+2}{2 \tau} \nabla R_{\phi} \cdot \nabla f-\frac{\tau-1}{\tau} \alpha\left(\tau_{\mathrm{g}} \phi\right)^{2}-\frac{\tau-1}{\tau}\left|\mathrm{Ric}_{\phi}-\frac{1}{m} R_{\phi} \mathrm{g}\right|^{2} \\
& -\frac{m+\tau-1}{m \tau}\left(R_{\phi}-m \lambda\right)\left(R_{\phi}-\frac{m(m-1)}{m+\tau-1} \lambda\right)
\end{aligned}
$$


where

$$
\operatorname{Ric}_{\phi}=\operatorname{Ric}-\alpha \nabla \phi \otimes \nabla \phi
$$

and

$$
R_{\phi}=\operatorname{trace}\left(\operatorname{Ric}_{\phi}\right)=R-\alpha|\nabla \phi|^{2} .
$$

Moreover, there exists a constant $\mu$ such that

$$
\begin{gathered}
R_{\phi}+\frac{\tau-1}{\tau}|\nabla f|^{2}-(m-\tau) \lambda=\mu e^{\frac{2}{\tau} f}, \\
\triangle f-|\nabla f|^{2}-\tau \lambda+\mu e^{\frac{2}{\tau} f}=0 .
\end{gathered}
$$

Proof. By the contracted second Bianchi identity

$$
\nabla R=2 \operatorname{div} \text { Ric, }
$$

the fact that

$$
\nabla|\nabla \phi|^{2}=2 \nabla_{\nabla \phi} \nabla \phi
$$

and

we have that

$$
\operatorname{div}(\nabla \phi \otimes \nabla \phi)=\tau_{\mathrm{g}} \phi \nabla \phi+\nabla_{\nabla \phi} \nabla \phi
$$

$$
\begin{aligned}
\nabla R_{\phi} & =\nabla R-\alpha \nabla|\nabla \phi|^{2}=2 \operatorname{div} \operatorname{Ric}-2 \alpha \nabla_{\nabla \phi} \nabla \phi \\
& =2 \operatorname{div} \operatorname{Ric}-2 \alpha\left(\operatorname{div}(\nabla \phi \otimes \nabla \phi)-\tau_{\mathrm{g}} \phi \nabla \phi\right) \\
& =2 \operatorname{div} \operatorname{Ric}_{\phi}+2 \alpha \tau_{\mathrm{g}} \phi \nabla \phi .
\end{aligned}
$$

Using the first equation in (1.6) and the identity [6]

$$
\operatorname{div} \operatorname{Hess} f=\operatorname{Ric} \nabla f+\nabla \triangle f,
$$

we have

$$
\begin{aligned}
\nabla R_{\phi} & =2 \operatorname{div}\left(\lambda \mathrm{g}+\frac{1}{\tau} \mathrm{d} f \otimes \mathrm{d} f-\operatorname{Hess} f\right)+2 \alpha \tau_{\mathrm{g}} \phi \nabla \phi \\
& =-2 \operatorname{div} \operatorname{Hess} f+\frac{2}{\tau} \triangle f \nabla f+\frac{2}{\tau} \nabla_{\nabla f} \nabla f+2 \alpha \tau_{\mathrm{g}} \phi \nabla \phi \\
& =-2\left(\operatorname{Ric}_{\phi} \nabla f+\nabla \triangle f\right)+\frac{2}{\tau} \triangle f \nabla f+\frac{2}{\tau} \nabla_{\nabla f} \nabla f,
\end{aligned}
$$

here in the last equality we have used the second equation in (1.6). Tracing the first equation in (1.6) yields

$$
R_{\phi}+\triangle f-\frac{1}{\tau}|\nabla f|^{2}=m \lambda .
$$

Plugging (2.9) into (2.8) leads to

$$
\nabla R_{\phi}=2 \operatorname{Ric}_{\phi} \nabla f+\frac{2}{\tau} \nabla_{\nabla f} \nabla f-\frac{2}{\tau} \triangle f \nabla f .
$$

From the first equation in (1.6), we have that

$$
\operatorname{Ric}_{\phi} \nabla f+\nabla_{\nabla f} \nabla f-\frac{1}{\tau}|\nabla f|^{2} \nabla f=\lambda \nabla f .
$$

Plugging this equality into (2.10) leads to

$$
\frac{1}{2} \nabla R_{\phi}=\frac{\tau-1}{\tau} \operatorname{Ric}_{\phi} \nabla f+\frac{1}{\tau}\left(R_{\phi}-(m-1) \lambda\right) \nabla f .
$$


Hence

$$
\frac{1}{2} \triangle R_{\phi}=\frac{\tau-1}{\tau} \operatorname{div}\left(\operatorname{Ric}_{\phi} \nabla f\right)+\frac{1}{\tau} \operatorname{div}\left(\left(R_{\phi}-(m-1) \lambda\right) \nabla f\right) .
$$

By (2.6) and (2.7) we have

$$
\begin{aligned}
& \operatorname{div}\left(\operatorname{Ric}_{\phi} \nabla f\right)=\left(\operatorname{div}_{\operatorname{Ric}_{\phi}}\right) \cdot \nabla f+\operatorname{trace}\left(\operatorname{Ric}_{\phi} \circ \operatorname{Hess} f\right) \\
& =\left(\frac{1}{2} \nabla R-\alpha\left(\tau_{\mathrm{g}} \phi \nabla \phi+\nabla_{\nabla \phi} \nabla \phi\right)\right) \cdot \nabla f \\
& +\operatorname{trace}\left(\operatorname{Ric}_{\phi} \circ\left(\frac{1}{\tau} \mathrm{d} f \otimes \mathrm{d} f+\lambda \mathrm{g}-\operatorname{Ric}_{\phi}\right)\right) \\
& =\frac{1}{2} \nabla R_{\phi} \cdot \nabla f-\alpha\left(\tau_{\mathrm{g}} \phi\right)^{2}+\operatorname{trace}\left(\operatorname{Ric}_{\phi} \circ\left(\lambda \mathrm{g}-\operatorname{Ric}_{\phi}\right)\right) \\
& +\frac{1}{\tau} \operatorname{Ric}_{\phi}(\nabla f, \nabla f) \text {. }
\end{aligned}
$$

By (2.11) we have

$$
\frac{1}{2} \nabla R_{\phi} \cdot \nabla f=\frac{\tau-1}{\tau} \operatorname{Ric}_{\phi}(\nabla f, \nabla f)+\frac{1}{\tau}\left(R_{\phi}-(m-1) \lambda\right)|\nabla f|^{2} .
$$

Plugging (2.14) into (2.13) leads to

$$
\begin{aligned}
\operatorname{div}\left(\operatorname{Ric}_{\phi} \nabla f\right)= & \frac{\tau}{2(\tau-1)} \nabla R_{\phi} \cdot \nabla f-\alpha\left(\tau_{\mathrm{g}} \phi\right)^{2}+\operatorname{trace}\left(\operatorname{Ric}_{\phi} \circ\left(\lambda \mathrm{g}-\operatorname{Ric}_{\phi}\right)\right) \\
& -\frac{1}{\tau(\tau-1)}\left(R_{\phi}-(m-1) \lambda\right)|\nabla f|^{2} .
\end{aligned}
$$

Plugging (2.15) into (2.12), we get

$$
\begin{aligned}
\frac{1}{2} \triangle R_{\phi}= & \frac{\tau+2}{2 \tau} \nabla R_{\phi} \cdot \nabla f-\frac{\tau-1}{\tau} \alpha\left(\tau_{\mathrm{g}} \phi\right)^{2} \\
& +\frac{\tau-1}{\tau} \operatorname{trace}\left(\operatorname{Ric}_{\phi} \circ\left(\lambda \mathrm{g}-\operatorname{Ric}_{\phi}\right)\right)-\frac{1}{\tau}\left(\mathrm{R}_{\phi}-(m-1) \lambda\right)\left(R_{\phi}-m \lambda\right) .
\end{aligned}
$$

It is easy to verify that

$$
\operatorname{trace}\left(\operatorname{Ric}_{\phi} \circ\left(\lambda \mathrm{g}-\operatorname{Ric}_{\phi}\right)\right)=-\left|\operatorname{Ric}_{\phi}-\frac{1}{m} R_{\phi} \mathrm{g}\right|^{2}+R_{\phi}\left(\lambda-\frac{1}{m} R_{\phi}\right) .
$$

Plugging (2.17) into (2.16), we arrive at (2.1).

For (2.4), by (2.11) we have

$$
\nabla\left(R_{\phi}+\frac{\tau-1}{\tau}|\nabla f|^{2}\right)-\frac{2}{\tau}\left(R_{\phi}+\frac{\tau-1}{\tau}|\nabla f|^{2}\right) \nabla f+\frac{2(m-\tau) \lambda}{\tau} \nabla f=0 .
$$

This identity can be rewritten as

$$
\nabla\left[e^{-\frac{2}{\tau} f}\left(R_{\phi}+\frac{\tau-1}{\tau}|\nabla f|^{2}\right)-(m-\tau) \lambda e^{-\frac{2}{\tau} f}\right]=0 .
$$

Hence there exists a constant $\mu$ so that

$$
e^{-\frac{2}{\tau} f}\left(R_{\phi}+\frac{\tau-1}{\tau}|\nabla f|^{2}\right)-(m-\tau) \lambda e^{-\frac{2}{\tau} f}=\mu,
$$

which is (2.4). From (2.4) and (2.9), we arrive at (2.5).

By a discussion similar to the proof of Theorem 2.1, we can derive formulas for the gradient Ricci-harmonic soliton metric. Similar formulas for the gradient Ricci soliton metric can be found in [11]. 
Theorem 2.2. Let $\mathrm{g}$ be a gradient Ricci-harmonic soliton metric defined in Definition 1.1. Then one can get

$$
\frac{1}{2} \triangle R_{\phi}=\frac{1}{2} \nabla R_{\phi} \cdot \nabla f-\alpha\left(\tau_{\mathrm{g}} \phi\right)^{2}-\left|\operatorname{Ric}_{\phi}-\frac{1}{m} R_{\phi} \mathrm{g}\right|^{2}-\frac{1}{m}\left(R_{\phi}-m \lambda\right) R_{\phi}
$$

where $\mathrm{Ric}_{\phi}$ and $R_{\phi}$ are defined in (2.2) and (2.3) respectively. Moreover, when $\lambda \neq 0$, by adding some constant on $f$, we have

$$
\begin{gathered}
R_{\phi}+|\nabla f|^{2}-2 \lambda f=0, \\
\triangle f-|\nabla f|^{2}+2 \lambda f=m \lambda .
\end{gathered}
$$

When $\lambda=0$, for some constant $\mu$, we have

$$
\begin{gathered}
R_{\phi}+|\nabla f|^{2}=\mu, \\
\triangle f-|\nabla f|^{2}=-\mu .
\end{gathered}
$$

Proof. Similar to (2.8), we have

$$
\begin{aligned}
\nabla R_{\phi} & =2 \operatorname{div}(\lambda \mathrm{g}-\operatorname{Hess} f)+2 \alpha \tau_{\mathrm{g}} \phi \nabla \phi \\
& =-2 \operatorname{div} \operatorname{Hess} f+2 \alpha \tau_{\mathrm{g}} \phi \nabla \phi=-2\left(\operatorname{Ric}_{\phi} \nabla f+\nabla \triangle f\right),
\end{aligned}
$$

here in the last equality we have used the second equation in (1.3). Tracing the first equation in (1.3) yields

$$
R_{\phi}+\triangle f=m \lambda
$$

Plugging (2.24) into (2.23) leads to

$$
\nabla R_{\phi}=2 \operatorname{Ric}_{\phi} \nabla f .
$$

Hence

$$
\frac{1}{2} \triangle R_{\phi}=\operatorname{div}\left(\operatorname{Ric}_{\phi} \nabla f\right)
$$

Similar to (2.13) we have

$$
\operatorname{div}\left(\operatorname{Ric}_{\phi} \nabla f\right)=\frac{1}{2} \nabla R_{\phi} \cdot \nabla f-\alpha\left(\tau_{\mathrm{g}} \phi\right)^{2}+\operatorname{trace}\left(\operatorname{Ric}_{\phi} \circ\left(\lambda \mathrm{g}-\operatorname{Ric}_{\phi}\right)\right) .
$$

Hence

$$
\frac{1}{2} \triangle R_{\phi}=\frac{1}{2} \nabla R_{\phi} \cdot \nabla f-\alpha\left(\tau_{\mathrm{g}} \phi\right)^{2}+\operatorname{trace}\left(\operatorname{Ric}_{\phi} \circ\left(\lambda \mathrm{g}-\operatorname{Ric}_{\phi}\right)\right) .
$$

Plugging (2.17) into (2.26), we arrive at (2.18).

For (2.19), by (2.25) we have

$$
\nabla\left(R_{\phi}+|\nabla f|^{2}\right)-2 \lambda \nabla f=0 .
$$

When $\lambda \neq 0$, by adding some constant on $f$, we get (2.19). From (2.19) and (2.24), we arrive at (2.20). When $\lambda=0$, there exists a constant $\mu$, such that (2.21) holds. From (2.21) and (2.24), we arrive at (2.22). 


\section{Rigid properties}

In this section, we study rigid properties by using the maximum principle or the weak maximum principle at infinity.

Theorem 3.1. Let $\mathrm{g}$ be a gradient Ricci-harmonic soliton metric on a compact manifold $M$.

1) If $\lambda>0$, then $R_{\phi} \geq 0$, i.e., $R \geq \alpha|\nabla \phi|^{2}$.

2) Any expanding or steady gradient Ricci-harmonic soliton metric should be trivial in the sense that $f$ is constant. Now the metric is harmonic Einstein (see (1.4) for the definition of the harmonic Einstein metric.)

Proof. Since $\alpha>0$, from (2.18) we have

$$
\frac{1}{2} \triangle R_{\phi} \leq \frac{1}{2} \nabla R_{\phi} \cdot \nabla f-\frac{1}{m}\left(R_{\phi}-m \lambda\right) R_{\phi} .
$$

By the maximum principle, we know that the minimal value of $R_{\phi}$ on $M$ satisfies

$$
\left(R_{\phi, \operatorname{mim}}-m \lambda\right) R_{\phi, \min } \leq 0,
$$

which implies that $R_{\phi, \text { mim }} \geq 0$ when $\lambda>0$, and $R_{\phi, \operatorname{mim}} \geq m \lambda$ when $\lambda \leq 0$.

We assume that $\lambda \leq 0$. Above discussion tells us that $R_{\phi} \geq m \lambda$, from (2.24) we know that $\triangle f \leq 0$. The Hopf's maximum principle tells us that $f$ is a constant function.

Remark 3.2. Part 2) of Theorem 3.1 was previously proved both by Müller [24] when introducing Ricci-Harmonic metrics, as well as by Williams [42].

Theorem 3.3. Let $\mathrm{g}$ be a $\tau$-quasi Ricci-harmonic metric on a compact manifold $M$. We also assume that $\tau \geq 1$.

1) If $\lambda \geq 0$, then

$$
R_{\phi} \geq \frac{m(m-1)}{m+\tau-1} \lambda
$$

i.e.,

$$
R \geq \alpha|\nabla \phi|^{2}+\frac{m(m-1)}{m+\tau-1} \lambda .
$$

2) If $\lambda \leq 0$, then $R_{\phi} \geq m \lambda$, i.e., $R \geq \alpha|\nabla \phi|^{2}+m \lambda$.

Proof. Since $\alpha>0$ and $\tau>1$, from (2.1) we have

$$
\frac{1}{2} \triangle R_{\phi} \leq \frac{\tau+2}{2 \tau} \nabla R_{\phi} \cdot \nabla f-\frac{m+\tau-1}{m \tau}\left(R_{\phi}-m \lambda\right)\left(R_{\phi}-\frac{m(m-1)}{m+\tau-1} \lambda\right) .
$$

By the maximum principle, we know that the minimal value of $R_{\phi}$ on $M$ satisfies

$$
\left(R_{\phi, \operatorname{mim}}-m \lambda\right)\left(R_{\phi, \operatorname{mim}}-\frac{m(m-1)}{m+\tau-1} \lambda\right) \leq 0,
$$

which implies that

$$
R_{\phi, \min } \geq \frac{m(m-1)}{m+\tau-1} \lambda,
$$

when $\lambda \geq 0$ and $R_{\phi, \min } \geq m \lambda$ when $\lambda \leq 0$. 
Remark 3.4. It was proved in [6] that the scalar curvature of a compact $\tau$-quasi Einstein metric satisfies

$$
R \geq \frac{m(m-1)}{m+\tau-1} \lambda
$$

Theorem 3.5. 1) Any $\tau$-quasi Ricci-harmonic metric with $\lambda \leq 0$ on a compact manifold $M$ should be trivial in the sense that $f$ is a constant function.

2) Any $\tau$-quasi Ricci-harmonic metric with $\lambda>0$ on a compact manifold should be trivial if $\mu \leq 0$, here $\mu$ is the constant in (2.4).

Proof. When $\lambda \neq 0,(2.5)$ can be rewritten as

$$
\triangle\left(e^{-\frac{2}{\tau} f}-\frac{\mu}{\tau \lambda}\right)=\frac{2-\tau}{\tau} e^{\frac{2}{\tau} f}\left|\nabla\left(e^{-\frac{2}{\tau} f}-\frac{\mu}{\tau \lambda}\right)\right|^{2}-2 \lambda\left(e^{-\frac{2}{\tau} f}-\frac{\mu}{\tau \lambda}\right) .
$$

When $\lambda<0$, the maximum principle tells us that $e^{-\frac{2}{\tau} f}-\frac{\mu}{\tau \lambda}$ is a constant function. When $\lambda>0$ and $\mu \leq 0$, from (3.3) we get that

$$
\triangle\left(e^{-\frac{2}{\tau} f}-\frac{\mu}{\tau \lambda}\right) \leq \frac{2-\tau}{\tau} e^{\frac{2}{\tau} f}\left|\nabla\left(e^{-\frac{2}{\tau} f}-\frac{\mu}{\tau \lambda}\right)\right|^{2} .
$$

By the Hopf's maximum principle we know that $e^{-\frac{2}{\tau} f}-\frac{\mu}{\tau \lambda}$ is a constant function. When $\lambda=0$, integrating (2.5) against measure $e^{-f} \mathrm{~d} V_{\mathrm{g}}$, we get

$$
\mu \int_{M} e^{\frac{(2-\tau) f}{\tau}} \mathrm{d} V_{\mathrm{g}}=-\int_{M} \operatorname{div}\left(e^{-f} \nabla f\right) \mathrm{d} V_{\mathrm{g}}=0
$$

which implies that $\mu=0$. Then (2.5) becomes $\triangle_{f} f=0$ and $f$ is a constant function.

Remark 3.6. Note that a $\tau$-quasi Ricci-harmonic metric defined in Definition 1.2 satisfies $\operatorname{Ric}_{f, \tau} \geq \lambda \mathrm{g}$. Hence a manifold with a $\tau-(\tau$ is finite $)$ quasi Ricci-harmonic metric is automatically compact when $\lambda>0$ [31]. Lü-Page-Pope [20] constructed nontrivial $\tau$-quasi Einstein metrics with $\lambda>0$ and $\tau>1$, which also satisfy $\mu>0$.

When considering rigid properties on noncompact manifolds, we need using the weak maximum principle at infinity. Recall that the weak maximum principle at infinity for $\triangle_{f}$ holds if for a given $\mathrm{C}^{2}$ function $u, \sup _{M} u=u^{*}<+\infty$, there exists a sequence $\left\{x_{n}\right\} \subset M$, such that $u\left(x_{n}\right)>u^{*}-\frac{1}{n}$, and $\triangle_{\mu} u\left(x_{n}\right) \leq \frac{1}{n}$. The following Lemma was given in [32].

Lemma 3.7. Let $(M, \mathrm{~g})$ be a complete weighted manifold satisfying the volume growth condition

$$
\int_{1}^{\infty} \frac{r}{\log \mu\left(B_{O}(r)\right)} \mathrm{d} r=+\infty
$$

where $\mu\left(B_{O}(r)\right)$ denote the weighted measure of a geodesic ball with radius $r$. Then the weak maximum principle at infinity for the weighted Laplacian $\triangle_{f}$ holds on $M$.

Lemma 3.8. The weak maximum principle at infinity for $\triangle_{f}$ holds on a complete manifold $M$ with metric g satisfying the coupled Ricci-harmonic systems (1.3) or (1.6).

Proof. The weighted volume comparison theorem established in [41] states that if the $\infty$-Bakry-Émery curvature of a smooth metric measure space $(M, \mathrm{~g}, \mathrm{~d} \mu=$ $\left.e^{-f} \mathrm{~d} V\right)$ satisfies $\operatorname{Ric}_{f} \geq \lambda$ for some constant $\lambda$, then for some fixed $R_{0}>0$, there 
exist constants $A, B, C>0$ such that for all $r \geq R_{0}$,

$$
\mu\left(B_{O}(r)\right) \leq A+B \int_{R_{0}}^{r} e^{-\lambda t^{2}+C t} \mathrm{~d} t .
$$

Obviously, (3.5) is valid for Ricci-harmonic metrics defined by (1.3) or (1.6). Then Lemma 3.7 tells us that the weak maximum principle at infinity for $\triangle_{f}$ holds.

After almost the same discussions as in [33] and [36], we can prove the following rigid properties.

Theorem 3.9. $\quad 1)$ Let g be an expanding or steady gradient Ricci-harmonic soliton metric on a complete noncompact manifold $M$. If

$$
\sup _{x \in M}\left\{|\nabla f|^{2}(x)\right\}<\infty
$$

then $f$ is a constant function and $\mathrm{g}$ is a harmonic Einstein metric.

2) Let g be a $\tau$-quasi Ricci-harmonic metric with $\lambda<0$ on a complete noncompact manifold $M$. If

$$
\sup _{x \in M}\left\{|\nabla f|^{2}(x)\right\}<-\frac{\tau^{2} \lambda}{m+\tau},
$$

then $f$ is a constant function and $g$ is a harmonic Einstein metric.

Proof. By Lemma 3.8, there exists a sequence $\left\{x_{k}\right\} \subset M$, such that

$$
|\nabla f|^{2}\left(x_{k}\right) \geq \sup _{M}|\nabla f|^{2}-\frac{1}{k}
$$

and

$$
\triangle_{f}|\nabla f|^{2}\left(x_{k}\right) \leq \frac{1}{k}
$$

Proof of 1). By the weighted Bochner's formula (1.2), the first equation in (1.3) and (2.20), we have

$$
\begin{aligned}
\frac{1}{2} \triangle_{f}|\nabla f|^{2} & =|\operatorname{Hess} f|^{2}+\nabla \triangle_{f} \cdot \nabla f+\operatorname{Ric}_{f}(\nabla f, \nabla f) \\
& =|\operatorname{Hess} f|^{2}-\lambda|\nabla f|^{2}+\alpha|\nabla \phi \cdot \nabla f|^{2} .
\end{aligned}
$$

If $\lambda<0$, from (3.8) we have that at $x_{k}$,

$$
\frac{1}{2 k} \geq \frac{1}{2} \triangle_{f}|\nabla f|^{2}\left(x_{k}\right) \geq-\lambda|\nabla f|^{2} \geq-\lambda\left(\sup _{M}|\nabla f|^{2}-\frac{1}{k}\right) .
$$

Letting $k \rightarrow \infty$ leads to $\sup _{M}|\nabla f|^{2} \leq 0$, which implies that $f$ is a constant function. If $\lambda=0$, by (2.20) and (3.8), we have

$$
\frac{1}{2} \triangle_{f}|\nabla f|^{2} \geq \frac{1}{m}(\triangle f)^{2}=\frac{1}{m}|\nabla f|^{4} .
$$

Hence

$$
\frac{1}{2 k} \geq \frac{1}{m}\left(\sup _{M}|\nabla f|^{2}-\frac{1}{k}\right)^{2} .
$$

Letting $k \rightarrow \infty$ leads to $\sup _{M}|\nabla f|^{2} \leq 0$, which implies that $f$ is a constant function. 
Proof of 2). By the weighted Bochner's formula (1.2), the first equation in (1.6) and (2.5), we have

$$
\begin{aligned}
\frac{1}{2} \triangle_{f}|\nabla f|^{2} & =|\operatorname{Hess} f|^{2}+\nabla \triangle_{f} \cdot \nabla f+\operatorname{Ric}_{f, \tau}(\nabla f, \nabla f)+\frac{1}{\tau}|\nabla f|^{4} \\
& \geq \frac{1}{m}(\triangle f)^{2}-\frac{2 \mu}{\tau} e^{\frac{2 f}{\tau}}|\nabla f|^{2}-\lambda|\nabla f|^{2}+\alpha|\nabla \phi \cdot \nabla f|^{2}+\frac{1}{\tau}|\nabla f|^{4} \\
& \geq \frac{1}{m}\left(|\nabla f|^{2}+\tau \lambda-\mu e^{\frac{2 f}{\tau}}\right)^{2}-\frac{2 \mu}{\tau} e^{\frac{2 f}{\tau}}|\nabla f|^{2}-\lambda|\nabla f|^{2}+\frac{1}{\tau}|\nabla f|^{4} \\
& \geq-\frac{m+\tau}{\tau^{2}}|\nabla f|^{4}-\lambda|\nabla f|^{2} .
\end{aligned}
$$

Then at $x_{k}$,

$$
-\frac{m+\tau}{\tau^{2}}|\nabla f|^{4}\left(x_{k}\right)-\lambda|\nabla f|^{2}\left(x_{k}\right) \leq \frac{1}{2 k} .
$$

By (3.7) we conclude that for $k$ large enough,

$$
\sup _{M}|\nabla f|^{2}-\frac{1}{k} \leq|\nabla f|^{2}\left(x_{k}\right) \leq \frac{-\lambda \tau^{2}+\sqrt{\tau^{4} \lambda^{2}-\frac{2}{k} \tau^{2}(m+\tau)}}{2(m+\tau)} .
$$

Letting $k \rightarrow \infty$ leads to $\sup _{M}|\nabla f|^{2} \leq 0$, which implies that $f$ is a constant function.

\section{Scalar curvature estimates}

Lower bound estimates for the scalar curvature on complete noncompact manifolds play important roles in the study of geometric properties of the Ricci-harmonic metric. We firstly give the scalar curvature estimates for the gradient Ricci-harmonic soliton metric.

Theorem 4.1. Let g be a gradient Ricci-harmonic soliton metric on a complete noncompact manifold $M$.

1) If $\lambda \geq 0$, then $R_{\phi} \geq 0$, i.e., $R \geq \alpha|\nabla \phi|^{2}$.

2) If $\lambda \leq 0$, then $R_{\phi} \geq m \lambda$, i.e., $R \geq \alpha|\nabla \phi|^{2}+m \lambda$. Moreover, $R_{\phi}>m \lambda$ unless $g$ is a harmonic Einstein metric and the gradient Ricci-harmonic soliton metric is trivial.

Proof. We firstly assume that $\lambda \geq 0$. Consider a smooth function $\theta(t)$ : $[0,+\infty) \rightarrow[0,1]$

$$
\theta(t)= \begin{cases}1, & 0 \leq t \leq 1 \\ 0, & t \geq 2\end{cases}
$$

so that

$$
-10 \theta^{\frac{1}{2}} \leq \theta^{\prime} \leq 0, \theta^{\prime \prime} \geq-10 .
$$

For some constant $R_{0}>1$ large enough, define smooth cutoff function $\varphi: M \rightarrow \mathbf{R}$ by $\varphi(x)=\theta\left(\frac{r(x)}{R_{0}}\right)$, where $r(x)=\operatorname{dist}(O, x)$ is the distance function determined by a fixed point $O \in M$. Then $\nabla \varphi=\frac{\theta^{\prime} \nabla r}{R_{0}}$. Theorem 2.1 in [41] states that if the Bakry-Émery curvature satisfies

$$
\operatorname{Ric}_{f}=\operatorname{Ric}+\operatorname{Hess} f \geq K \mathrm{~g}
$$


for some constant $K \geq 0$, then for any given minimal geodesic segment $\gamma(t)$ and $r_{0}>0$,

$$
\left(\triangle_{f} r\right)(\gamma(t)) \leq\left(\triangle_{f} r\right)\left(\gamma\left(r_{0}\right)\right)-K\left(t-r_{0}\right)
$$

holds for all $t \geq r_{0}$. Let

$$
D=\sup _{x \in \partial B_{O}(1)}\left|\left(\triangle_{f} r\right)(x)\right| .
$$

We then get that $\left(\triangle_{f} r\right)(r(x)) \leq D$ for a shrinking or steady gradient Ricci-harmonic soliton metric when $r(x) \geq 1$. Hence for $y \in B_{O}\left(2 R_{0}\right)$,

$$
\triangle_{f} \varphi(y)=\triangle \varphi-\nabla \varphi \cdot \nabla f=\frac{\theta^{\prime \prime}}{R_{0}^{2}}+\frac{\theta^{\prime} \triangle_{f} r}{R_{0}} \geq \frac{\theta^{\prime \prime}}{R_{0}^{2}}+\frac{D \theta^{\prime}}{R_{0}}
$$

Let $Q=R_{\phi} \varphi$. We will finish the proof by contradiction. Assume that for some $R_{0}$ large enough, the minimal value of $R_{\phi}$ on $B_{O}\left(R_{0}\right)$ is negative. We also assume that the minimal value of $Q$ on $B_{O}\left(2 R_{0}\right)$ is achieved at $x_{0}$. Then $\varphi\left(x_{0}\right)>0, \nabla Q\left(x_{0}\right)=0$ and $\triangle_{f} Q\left(x_{0}\right) \geq 0$. In the following we will estimate at $x_{0}$. Note that

$$
\nabla R_{\phi}=-\frac{Q \nabla \varphi}{\varphi^{2}}
$$

Hence

$$
\triangle_{f} Q=\frac{\triangle_{f} \varphi}{\varphi} Q-\frac{2|\nabla \varphi|^{2} Q}{\varphi^{2}}+\varphi \triangle_{f} R_{\phi}
$$

By (4.3) and (4.4), we get

$$
0 \leq \triangle_{f} \varphi Q-\frac{2|\nabla \varphi|^{2} Q}{\varphi}-\frac{Q-m \lambda \varphi}{m} Q \leq-\frac{210+10 D R_{0}}{R_{0}^{2}} Q-\frac{Q-m \lambda \varphi}{m} Q .
$$

Hence

$$
Q \geq-\frac{210+10 D R_{0}}{R_{0}^{2}} m+m \lambda \varphi
$$

Let

Since

$$
\sigma\left(R_{0}\right)=\frac{\inf _{B_{O}\left(R_{0}\right)} R_{\phi}}{\inf _{B_{O}\left(2 R_{0}\right)} R_{\phi}} .
$$

We have that

$$
\varphi\left(x_{0}\right) \inf _{B_{O}\left(2 R_{0}\right)} R_{\phi} \leq \varphi\left(x_{0}\right) R_{\phi}\left(x_{0}\right) \leq \inf _{B_{O}\left(R_{0}\right)} R_{\phi} .
$$

Hence for all $x \in B_{O}\left(R_{0}\right)$,

$$
R_{\phi}(x) \geq Q\left(x_{0}\right) \geq-\frac{210+10 D R_{0}}{R_{0}^{2}} m+m \lambda \sigma\left(R_{0}\right),
$$

which implies that $R_{\phi}$ is bounded from below. Then $\sigma\left(R_{0}\right) \rightarrow 1$ as $R_{0} \rightarrow \infty$. Letting $R_{0} \rightarrow \infty$, we have $R_{\phi} \geq m \lambda \geq 0$, which contradicted to the assumption. Hence $R_{\phi} \geq 0$.

Now we assume that $\lambda<0$. In this case, the method of our proof comes from [33]. Rewrite (3.1) as

$$
\frac{1}{2} \triangle_{f} R_{\phi} \leq-\frac{1}{m} R_{\phi}^{2}+\lambda R_{\phi}
$$

From the volume growth estimate (3.5) and Corollary 13 in [33], we get that

$$
R_{\phi, \text { inf }}=\inf _{x \in M}\left\{R_{\phi}(x)\right\}>-\infty
$$


Lemma 3.8 tells us that there exists a sequence $\left\{x_{k}\right\} \subset M$ such that

$$
R_{\phi, \text { inf }} \leq R_{\phi}\left(x_{k}\right)<R_{\phi, \text { inf }}+\frac{1}{k}, \quad \triangle_{f}\left(R_{\phi}\right)\left(x_{k}\right) \geq-\frac{1}{k} .
$$

Setting $x=x_{k}$ in (4.6) and letting $k \rightarrow \infty$, we conclude that

$$
-\frac{1}{m}\left(R_{\phi, \mathrm{inf}}\right)^{2}+\lambda R_{\phi, \mathrm{inf}} \geq 0
$$

which implies that $R_{\phi} \geq R_{\phi, \text { inf }} \geq m \lambda$. Now we assume that $R_{\phi}\left(x_{0}\right)=m \lambda<0$ for some $x_{0} \in M$. Note that $R_{\phi}-m \lambda$ satisfies

$$
\frac{1}{2} \triangle_{f}\left(R_{\phi}-m \lambda\right) \leq-\lambda\left(R_{\phi}-m \lambda\right)
$$

By the maximum principle [13] we know that $R_{\phi}-m \lambda \equiv 0$. Then (2.18) tells us that

$$
\operatorname{Ric}_{\phi}=\frac{1}{m} R_{\phi} \mathrm{g}=\lambda \mathrm{g}
$$

and $\tau_{\mathrm{g}} \phi=0$. Hence $\mathrm{g}$ is a harmonic Einstein metric.

Remark 4.2. Chen [8] proved that the scalar curvature of a steady or shrinking gradient Ricci soliton metric is nonnegative. The scalar curvature of an expanding gradient Ricci soliton metric satisfies $R \geq m \lambda$, which was proved in [45].

Corollary 4.3. Let g be a nontrivial steady gradient Ricci-harmonic soliton metric on a complete noncompact manifold. Then the constant $\mu$, appeared in (2.21), is positive.

Proof. Part 1) of Theorem 4.1 tells us that $R_{\phi} \geq 0$. We deduce from (2.19) that $\mu \geq 0$. If $\mu=0$ then $|\nabla f|=0$. Hence $f$ is a constant function, which is impossible since $\mathrm{g}$ is nontrivial.

Corollary 4.4. Let g be an expanding nontrivial gradient Ricci-harmonic soliton metric on a complete noncompact manifold $M$. Then $f$ has no lower bound.

Proof. If we assume that for some constant $C>0, f \geq-C$. From (2.19) and Part 2) in Theorem 4.1, we have

$$
|\nabla f|^{2} \leq-m \lambda-2 C \lambda<+\infty
$$

Then Part 1) of Theorem 3.9 tells us that $\mathrm{g}$ is trivial. This is a contradiction and we finish the proof.

In the following we will study the scalar curvature estimate for the nontrivial complete noncompact $\tau$-quasi Ricci-harmonic metric. Firstly we establish a gradient estimate for $f$.

Lemma 4.5. Let g be a $\tau$-quasi Ricci-harmonic metric on a complete noncompact manifold $M$. If $\lambda \leq 0$ and $\mu \leq 0$, where $\mu$ is the constant in (2.4), then

$$
|\nabla f|^{2}(x) \leq-\tau \lambda
$$

holds for all $x \in M$. 
Proof. By the weighted Bochner's formula (1.2), (2.5) and the first equation in (1.6), we have

$$
\begin{aligned}
\frac{1}{2} \triangle_{f}|\nabla f|^{2} & =|\operatorname{Hess} f|^{2}+\operatorname{Ric}_{f}(\nabla f, \nabla f)+\nabla \triangle_{f} f \cdot \nabla f \\
& \geq \lambda|\nabla f|^{2}+\alpha|\nabla \phi \cdot \nabla f|^{2}+\frac{1}{\tau}|\nabla f|^{4}-\frac{2 \mu}{\tau} e^{\frac{2}{\tau} f}|\nabla f|^{2} \\
& \geq \lambda|\nabla f|^{2}+\frac{1}{\tau}|\nabla f|^{4} .
\end{aligned}
$$

Let $\varphi(x)=\theta\left(\frac{r(x)}{R_{0}}\right)$ be the cutoff function used in the proof of Theorem 4.1. The first equation in (1.6) tells us that

$$
\operatorname{Ric}_{f, \tau}=\alpha \nabla \phi \otimes \nabla \phi+\lambda \mathrm{g} \geq \lambda \mathrm{g} .
$$

By the weighted Laplacian comparison theorem [16, 35], we have that for all $x \in$ $B_{O}\left(2 R_{0}\right)$,

$$
\triangle_{f} \varphi(x)=\frac{\theta^{\prime \prime}}{R_{0}^{2}}+\frac{\theta^{\prime} \triangle_{f} r}{R_{0}} \geq \frac{\theta^{\prime \prime}}{R_{0}^{2}}+\frac{(m+\tau-1) \theta^{\prime}\left(1+\sqrt{-\frac{\lambda}{m+\tau-1}} R_{0}\right)}{R_{0}^{2}} .
$$

Let $P=\varphi|\nabla f|^{2}$. Assume that for $R_{0}$ large enough, the maximal value of $P$ on $B_{O}\left(2 R_{0}\right)$, achieved at $x_{0}$, is positive. Then $\varphi\left(x_{0}\right)>0, \nabla P\left(x_{0}\right)=0$ and $\triangle_{f} P\left(x_{0}\right) \leq 0$. By using (4.8) and (4.9), similar to the proof of Theorem 4.1, we have the following estimate at $x_{0}$,

$$
\frac{2}{\tau} P^{2}+\left[2 \lambda-\frac{210+10(m+\tau-1)+10 \sqrt{-(m+\tau-1) \lambda} R_{0}}{R_{0}^{2}}\right] P \leq 0 .
$$

Solving this inequality leads to

$$
P\left(x_{0}\right) \leq-\tau \lambda+\tau \frac{105+5(m+\tau-1)+5 \sqrt{-(m+\tau-1) \lambda} R_{0}}{R_{0}^{2}} .
$$

Hence for all $x \in B_{O}\left(R_{0}\right)$,

$$
|\nabla f|^{2}(x)=P(x) \leq P\left(x_{0}\right) \leq-\tau \lambda+\tau \frac{105+5(m+\tau-1)+5 \sqrt{-(m+\tau-1) \lambda} R_{0}}{R_{0}^{2}} .
$$

We arrive at (4.7) by letting $R_{0} \rightarrow \infty$ in the above inequality.

Corollary 4.6. Any $\tau$-quasi Ricci-harmonic metrics with $\lambda=0$ and $\mu \leq 0$ on complete noncompact manifolds should be trivial.

Due to Remark 3.6 and Corollary 4.6, we know that constants $\lambda$ and $\mu$ of a nontrivial $\tau$-quasi Ricci-harmonic metric on a noncompact manifold should satisfy $\lambda<0$, or $\lambda=0$ and $\mu>0$.

Theorem 4.7. Let $\mathrm{g}$ be a $\tau$-quasi Ricci-harmonic metric, with $\lambda<0$ or $\lambda=0$ and $\mu>0$, on a complete noncompact manifold $M$. Then

$$
R_{\phi}(x)=R(x)-\alpha|\nabla \phi|^{2}(x) \geq m \lambda
$$

holds for all $x \in M$.

Proof. We firstly assume that $\mu<0$ and $0<\tau \leq 1$. By using (2.5), we have

$$
\triangle_{f} e^{\frac{2}{\tau} f}=\frac{4}{\tau^{2}} e^{\frac{2}{\tau} f}|\nabla f|^{2}+\frac{2}{\tau} e^{\frac{2}{\tau} f} \triangle_{f} f \geq \frac{2}{\tau} e^{\frac{2}{\tau} f} \triangle_{f} f=\frac{2}{\tau} e^{\frac{2}{\tau} f}\left(\tau \lambda-\mu e^{\frac{2}{\tau} f}\right) .
$$


Let $S=\varphi e^{\frac{2}{\tau} f}$, where $\varphi(x)=\theta\left(\frac{r(x)}{R_{0}}\right)$ is the cutoff function used in the proof of Theorem 4.1. We assume that $S$ achieves its maximal value on $B_{O}\left(2 R_{0}\right)$ at $x_{0}$. Then $\varphi\left(x_{0}\right)>0, \nabla S\left(x_{0}\right)=0$ and $\triangle_{f} S\left(x_{0}\right) \leq 0$. By (4.9) and a standard discussion, we conclude that

$$
e^{\frac{2}{\tau} f} \leq \frac{\tau \lambda}{\mu}
$$

Plugging this estimate into (2.4) leads to (4.10). When $\mu \geq 0$ and $0<\tau \leq 1$, (4.10) directly comes from (2.4). When $\mu \leq 0$ and $\tau>1$, let $\varphi(x)=\theta\left(\frac{r(x)}{R_{0}}\right)$ be the cutoff function used above. Let $Q=R_{\phi} \varphi$. We assume that for $R_{0}$ large enough, the minimal value of $Q$ on $B_{O}\left(2 R_{0}\right)$, achieved at $x_{o}$, is negative. Hence at $x_{0}, \varphi>0, \nabla Q=0$ and $\triangle_{f} Q \geq 0$. Based on (2.1), we have that at $x_{0}$,

$$
\begin{aligned}
0 & \leq \triangle_{f} \varphi Q-\frac{2|\nabla \varphi|^{2}}{\varphi} Q-\frac{2 \nabla f \cdot \nabla \varphi}{\tau} Q-\frac{2(m+\tau-1)}{m \tau}(Q-m \lambda \varphi)\left(Q-\frac{m(m-1)}{m+\tau-1} \lambda \varphi\right) \\
& \leq \triangle_{f} \varphi Q-\frac{2|\nabla \varphi|^{2}}{\varphi} Q+\frac{2 C|\nabla \varphi|}{\tau} Q-\frac{2(m+\tau-1)}{m \tau}(Q-m \lambda \varphi)\left(Q-\frac{m(m-1)}{m+\tau-1} \lambda \varphi\right),
\end{aligned}
$$

where the second inequality comes from Lemma 4.5. After a standard discussion as above, we arrive at (4.10).

The only rest case is that $\mu>0$ and $\tau>1$. We use the method given in [37] and list the main steps as follows. Let

$$
G=R_{\phi}+2 t e^{\frac{2}{\tau} f}
$$

for $t>0$, and $\varphi(x)=\theta\left(\frac{r(x)}{R_{0}}\right)$ be the cutoff function used above. Set $H=G \varphi$. We assume that for $R_{0}>0$ large enough, the minimal value of $G$ on $B_{O}\left(R_{0}\right)$ is negative. We also assume that the minimal value of $H$ on $B_{O}\left(2 R_{0}\right)$ is achieved at $x_{0}$. Then at $x_{0}, \varphi>0, \nabla H=0$ and $\triangle_{f} H \geq 0$. By $(2.4)$ we get

$$
|\nabla f|^{2}=-\frac{\tau}{\tau-1} G+\frac{\tau(2 t+\mu)}{\tau-1} e^{\frac{2}{\tau} f}+\frac{\tau(m-\tau)}{\tau-1} \lambda .
$$

Note that

$$
\triangle_{f} e^{\frac{2}{\tau} f}=\left(\frac{4}{\tau^{2}}|\nabla f|^{2}+\frac{2}{\tau} \triangle_{f} f\right) e^{\frac{2}{\tau} f}
$$

By using (4.12), (4.13) and (4.2), we have that for all $\epsilon>0$,

$$
\begin{aligned}
\frac{1}{2} \triangle_{f} G \leq & \frac{\varphi}{4 \epsilon \tau}|\nabla G|^{2}+\frac{4 t(m+\tau-1)}{m \tau} G e^{\frac{2}{\tau} f}-\frac{m+\tau-1}{m \tau} G^{2} \\
& +\left(\frac{2 m-2+\tau}{\tau} \lambda-\frac{\epsilon}{(\tau-1) \varphi}\right) G-\frac{m(m-1)}{\tau} \lambda^{2}+\frac{m-\tau}{(\tau-1) \varphi} \epsilon \lambda \\
& -\frac{4 t^{2}(m+\tau-1)+2 t m \mu}{m \tau} e^{\frac{4}{\tau} f}-\left(\frac{4 t(m-1)}{\tau} \lambda-\frac{\epsilon(2 t+\mu)}{(\tau-1) \varphi}\right) e^{\frac{2}{\tau} f}
\end{aligned}
$$

holds at $x_{0}$. Since $G\left(x_{0}\right) \leq 0$, we have

$$
\begin{aligned}
\frac{1}{2} \triangle_{f} G \leq & \frac{\varphi}{4 \epsilon \tau}|\nabla G|^{2}-\frac{m+\tau-1}{m \tau} G^{2} \\
& +\left(\frac{2 m-2+\tau}{\tau} \lambda-\frac{\epsilon}{(\tau-1) \varphi}\right) G-\frac{m(m-1)}{\tau} \lambda^{2}+\frac{m-\tau}{(\tau-1) \varphi} \epsilon \lambda \\
& \frac{m \tau}{16 t^{2}(m+\tau-1)+8 m t \mu}\left(\frac{4 t(m-1)}{\tau} \lambda-\frac{\epsilon(2 t+\mu)}{(\tau-1) \varphi}\right)^{2} .
\end{aligned}
$$


Now a standard discussion as above shows that for all $x \in M$,

$$
G(x)=R_{\phi}(x)+2 t e^{\frac{2}{\tau} f(x)} \geq \frac{m(2 m-2+\tau)+m \sqrt{\tau^{2}+\frac{8(m+\tau-1)(m-1)^{2} t}{2 t(m+\tau-1)+m \mu}}}{2(m+\tau-1)} \lambda .
$$

We arrive at (4.10) by letting $t \searrow 0$ in (4.14).

\section{Potential function estimate}

Potential function estimates for the gradient Ricci soliton metric have been studied in literature. For example, Cao-Zhou [3] proved that the potential function of a noncompact shrinking gradient Ricci soliton metric with $\lambda=\frac{1}{2}$ should satisfies the following growth estimate

$$
\frac{1}{4}(r(x)-c)^{2} \leq f(x) \leq \frac{1}{4}(r(x)+c)^{2},
$$

where $r(x)$, the distance function determined by some fixed point $O \in M$, is large enough, and $c$ is a positive constant depending only on $m$ and the geometry of $g$ on the unit ball $B_{O}(1)$. Also in this paper, based on the estimate of the potential function, the authors proved that any gradient shrinking Ricci soliton metric has at most Euclidean volume growth. Cao-Zhou's result was generalized to the shrinking gradient Ricci-harmonic soliton metric in [44]. We state the generalized result as follows.

Theorem 5.1. Let g be a shrinking gradient Ricci-harmonic soliton metric on a complete noncompact manifold $M$. Then $f$ satisfies

$$
\frac{\lambda}{2}(r(x)-c)^{2} \leq f(x) \leq \frac{\lambda}{2}(r(x)+c)^{2},
$$

where $r(x)$ is large enough and $c>0$ is independent of $x$. Moreover, there exists some constant $C>0$ such that $V\left(B_{O}(r)\right) \leq C r^{n}$ holds for $r>0$ large enough.

Corollary 4.4 says that the potential function of any expanding nontrivial gradient Ricci-harmonic soliton metric on a complete noncompact manifold has no lower bound. The following theorem gives a specific estimate of $f$ and a growth estimate of the volume.

Theorem 5.2. Let g be an expanding gradient Ricci-harmonic soliton metric on a complete noncompact manifold $M$. Then $f$ satisfies

$$
-\frac{\lambda}{2} r^{2}-c r^{\frac{3}{2}} \sqrt{\ln r} \leq \sup _{x \in \partial\left(B_{O}(r)\right)}(-f)(x) \leq-\frac{\lambda}{2} r^{2}+c r,
$$

where $r(x)$ is large enough and $c>0$ is independent of $x$. Moreover, there exists some constant $C>0$ such that

$$
V\left(B_{O}(r)\right) \leq C e^{\sqrt{-2 \lambda(m-1)} r}
$$

for $r>0$ large enough.

Proof. We use the method given in [27] and list the main steps as follows. From (2.19) and Part 2) of Theorem 4.1, we have

$$
|\nabla f|=\sqrt{2 \lambda f-R_{\phi}} \leq \sqrt{2 \lambda f-m \lambda},
$$

or

$$
|\nabla \sqrt{2 \lambda f-m \lambda}| \leq-\lambda .
$$


Hence

$$
\sqrt{2 \lambda f(x)-m \lambda}-\sqrt{2 \lambda f(O)-m \lambda} \leq-\lambda r(x) .
$$

Then the second inequality in (5.2) follows.

Now we can prove volume growth estimate (5.3). Let $\left.\mathrm{d} V\right|_{\exp _{O}(r, \xi)}=J(r, \xi) \mathrm{d} r \mathrm{~d} \xi$ be the volume form in a normal coordinate centered at $O$ for $r>0$ and $\xi \in T_{O}(M)$. Then the Ricatti equation shows that along any minimizing geodesic starting from $O$,

$$
\left(\frac{J^{\prime}}{J}\right)^{\prime}(r)+\frac{1}{m-1}\left(\frac{J^{\prime}}{J}\right)^{2}(r)+\operatorname{Ric}\left(\partial_{r}, \partial_{r}\right) \leq 0 .
$$

In the following we use $c_{1}, c_{2}, \ldots$ to denote positive constants independent of $r$. Set $u(r)=\frac{J^{\prime}(r)}{J(r)}$. Integrating above inequality from 1 to $r>1$ and using the first equation in (1.3), we have

$$
u(r)+\frac{1}{m-1} \int_{1}^{r} u^{2}(s) \mathrm{d} s \leq f^{\prime}(r)-\lambda r+c_{1} \leq-2 \lambda r+c_{2},
$$

where we have used the fact that $|\nabla f|(x) \leq-\lambda r(x)+c_{3}$, which comes from (5.4) and the second inequality in (5.2). By the Cauchy-Schwarz inequality we have

$$
u(r)+\frac{1}{(m-1) r}\left(\int_{1}^{r} u(s) \mathrm{d} s\right)^{2} \leq-2 \lambda r+c_{2} .
$$

We claim that

$$
\int_{1}^{r} u(s) \mathrm{d} s \leq \sqrt{-2 \lambda(m-1)} r+\sqrt{-\frac{m-1}{2 \lambda}} c_{2} .
$$

To prove (5.6), for $r \geq 1$ we set

$$
v(r)=\sqrt{-2 \lambda(m-1)} r+\sqrt{-\frac{m-1}{2 \lambda}} c_{2}-\int_{1}^{r} u(s) \mathrm{d} s .
$$

Obviously $v(1)>0$. We assume that $v(r)$ does not remain positive for all $r \geq 1$ and let $R>1$ be the first number such that $v(R)=0$. Then

$$
\int_{1}^{R} u(s) \mathrm{d} s=\sqrt{-2 \lambda(m-1)} R+\sqrt{-\frac{m-1}{2 \lambda}} c_{2} .
$$

Hence

$$
\frac{1}{(m-1) R}\left(\int_{1}^{R} u(s) \mathrm{d} s\right)^{2} \geq-2 \lambda R+2 c_{2} .
$$

Plugging this inequality into (5.5) we conclude that $u(R) \leq-c_{2}<0$, which implies that $v^{\prime}(R)>0$, so there exists a small positive number $\delta$ such that $v(R-\delta)<v(R)=$ 0 . This contradicts with the choice of $R$. Hence (5.6) holds. Note that (5.6) can be rewritten as

$$
\ln J(r)-\ln J(1) \leq \sqrt{-2 \lambda(m-1)} r+\sqrt{-\frac{m-1}{2 \lambda}} c_{2} .
$$

We easily arrive at (5.3) from above inequality.

When we replace the scalar curvature to be $R_{\phi}$ and let $\lambda=-\frac{1}{2}$ in the proof of Theorem 5.1 in [27], we will get that for an expanding gradient Ricci-harmonic soliton metric with $\lambda=-\frac{1}{2}$,

$$
\sup _{x \in \partial\left(B_{O}(r)\right)}(-f)(x) \geq \frac{1}{4} r^{2}-c r^{\frac{3}{2}} \sqrt{\ln r} .
$$


Then the first inequality in (5.2) follows by rescaling of the metric.

Potential function estimates for the $\tau$-quasi Einstein metric were established in [40]. By using (2.4), (2.5) and the method used in [40], we can derive potential function estimates for the $\tau$-quasi Ricci-harmonic metric.

Theorem 5.3. Let g be a $\tau$-quasi Ricci-harmonic metric with $\tau>1$ on a complete noncompact manifold $M$. We use $c>0$ to denote some constant independent of $r>1$.

1) If $\lambda<0, \mu<0$, then for $r>1$,

$$
-\frac{\tau}{2} \ln \frac{\tau \lambda}{\mu} \leq \sup _{x \in \partial B_{O}(r)}(-f)(x) \leq \frac{\tau}{\sqrt{\tau-1}} \sqrt{-\lambda} r+c .
$$

2) If $\lambda<0$ and $\mu>0$, then for $r>1$

$$
\begin{aligned}
\frac{2 \tau}{\sqrt{m+5 \tau-1}+\sqrt{m+\tau-1}} \sqrt{-\lambda} r-c & \leq \sup _{x \in \partial B_{O}(r)}(-f)(x) \\
& \leq \frac{\tau}{\sqrt{\tau-1}} \sqrt{-\lambda} r+c .
\end{aligned}
$$

3) If $\lambda<0$ and $\mu=0$, then for $r>1$,

$$
\frac{\tau}{\sqrt{m+\tau-1}} \sqrt{-\lambda} r-c \leq \sup _{x \in \partial B_{O}(r)}(-f)(x) \leq \frac{\tau}{\sqrt{\tau-1}} \sqrt{-\lambda} r+c .
$$

4) If $\lambda=0$ and $\mu>0$, then for $r>1$,

$$
\frac{\tau-1}{m+\tau-1} \ln r-c \leq \sup _{x \in \partial B_{O}(r)}(-f)(x) \leq \tau \ln r+c .
$$

Proof. Since $R_{\phi} \geq m \lambda$, we deduce from (2.4) that

$$
|\nabla f|^{2} \leq \frac{\tau \mu}{\tau-1} e^{\frac{2}{\tau} f}-\frac{\tau^{2} \lambda}{\tau-1}
$$

Then the second inequalities in (5.7), (5.8), (5.9) and (5.10) can be deduced from above differential inequality easily after a same discussion as in the proof of (5.2). The first inequality in (5.7) comes from (4.11). Due to (2.4), (2.5), when we replace the scalar curvature in [40] by $R_{\phi}$, we will get the first inequalities in (5.8), (5.9) and (5.10).

\section{6. $L_{f}^{2}$-spectrum and diameter}

The bottom of the $L_{f}^{2}$-spectrum is defined by

$$
\lambda_{f}(M):=\inf _{\phi \in C_{0}^{\infty}(M)} \frac{\int_{M}|\nabla \phi|^{2} e^{-f} \mathrm{~d} V}{\int_{M} \phi^{2} e^{-f} \mathrm{~d} V}=\inf _{\phi \in C_{0}^{\infty}(M)} \frac{\int_{M}|\nabla \phi|^{2} \mathrm{~d} \mu}{\int_{M} \phi^{2} \mathrm{~d} \mu},
$$

and the bottom of the $L^{2}$-spectrum is defined by

$$
\lambda(M):=\inf _{\phi \in C_{0}^{\infty}(M)} \frac{\int_{M}|\nabla \phi|^{2} \mathrm{~d} V}{\int_{M} \phi^{2} \mathrm{~d} V} .
$$

There has been an active interest in the study of $\lambda(M)$ under conditions about the Ricci curvature. The upper bound estimate of $\lambda_{f}(M)$ was derived in [35] via the $\tau$-Bakry-Émery curvature. If we assume that the $(\infty$-)Bakry-Émery curvature is bounded from below and $f$ is of linear growth, we can also get the upper bound 
estimate of $\lambda_{f}(M)$ [27]. When we consider the lower bound estimate of $\lambda_{f}(M)$, the following lemma, established in [26], is useful.

Lemma 6.1. Let $(M, \mathrm{~g})$ be a complete noncompact Riemannian manifold and $f$ be a smooth function on $M$. If there exists a positive function $v>0$ such that $\triangle_{f} v \leq-\lambda v$ for some constant $\lambda>0$, then $\lambda_{f}(M) \geq \lambda$.

In [26], the authors proved that $\lambda_{f}(M)=\frac{\mu}{4}$ holds for a nontrivial steady gradient Ricci soliton metric, where $\mu>0$ appears in the equality

$$
R+|\nabla f|^{2}=\mu \text {. }
$$

With the similar discussion as in [26], we can get the following result.

Theorem 6.2. Let g be a nontrivial steady gradient Ricci-harmonic soliton metric on a complete noncompact manifold. Then

$$
\lambda_{f}(M)=\frac{\mu}{4}
$$

where $\mu$ is the constant in (2.21).

Proof. Since $R_{\phi} \geq 0$, from (2.21) we have that

$$
|\nabla f| \leq \sqrt{\mu}
$$

We also note that $\operatorname{Ric}_{f}=\alpha \nabla \phi \otimes \nabla \phi \geq 0$. Theorem 2.2 in [26] tells us that $\lambda_{f}(M) \leq \frac{\mu}{4}$. On the other hand, by (2.22) and (6.4) we have

$$
\triangle_{f} e^{\frac{f}{2}}=\frac{1}{2}\left(\triangle_{f} f+\frac{1}{2}|\nabla f|^{2}\right) e^{\frac{f}{2}}=\frac{1}{2}\left(-\mu+\frac{1}{2}|\nabla f|^{2}\right) e^{\frac{f}{2}} \leq-\frac{1}{4} \mu e^{\frac{f}{2}} .
$$

By Lemma 6.1 we get that $\lambda_{f}(M) \geq \frac{\mu}{4}$. Hence (6.3) holds.

The author [38] derived a gap estimate of $\lambda_{f}(M)$ for a noncompact $\tau$-quasi Einstein metric. By a similar discussion as in [38], we can prove the following gap estimate of $\lambda_{f}(M)$ for a noncompact $\tau$-quasi Ricci-harmonic metric.

Theorem 6.3. Let $\mathrm{g}$ be a $\tau$-quasi Ricci-harmonic metric with $\tau>1$ on a complete noncompact manifold $M$. If $\lambda \leq 0$ and $\mu \geq 0$, then

Proof. Since

$$
-\frac{\tau-1}{4} \lambda \leq \lambda_{f}(M) \leq-\frac{m+\tau-1}{4} \lambda .
$$

$$
\operatorname{Ric}_{f, \tau}=\lambda \mathrm{g}+\alpha \nabla \phi \otimes \nabla \phi \geq \lambda \mathrm{g} .
$$

The upper bound estimate of $\lambda_{f}(M)$ directly follows from [35, 43]. For the lower bound, we compute as follows

$$
\begin{aligned}
\triangle_{f} e^{\frac{\tau-1}{2 \tau} f} & =\left(\frac{(\tau-1)^{2}}{4 \tau^{2}}|\nabla f|^{2}+\frac{\tau-1}{2} \lambda-\frac{\tau-1}{2 \tau} \mu e^{\frac{2}{\tau} f}\right) e^{\frac{\tau-1}{2 \tau} f} \\
& \leq-\frac{\tau-1}{4 \tau}\left(\mu e^{\frac{2}{\tau} f}-\tau \lambda\right) e^{\frac{\tau-1}{2 \tau} f} \leq \frac{\tau-1}{4} \lambda e^{\frac{\tau-1}{2 \tau} f}
\end{aligned}
$$

where the second inequality comes from the fact that

$$
|\nabla f|^{2} \leq \frac{\tau}{\tau-1}\left(\mu e^{\frac{2}{\tau} f}-\tau \lambda\right)
$$

which follows by plugging (4.10) into (2.4). Hence the lower bound of $\lambda_{f}(M)$ comes from Lemma 6.1. 
Remark 6.4. In fact the upper bound estimate in (6.5) holds for $\tau>0$ and $\lambda \leq 0[35,43]$. We can deduced from Theorem 6.3 that $\lambda_{f}(M)=0$ holds for a $\tau$ quasi Ricci-harmonic metric with $\tau>1, \lambda=0$ and $\mu>0$ on a complete noncompact manifold.

Now we study the diameter estimate for the Ricci-harmonic metric on compact manifolds. Recall that there does not exist nontrivial expanding or steady gradient Ricci-harmonic soliton metric on any compact manifolds (see Theorem 3.1). A recent result in [34] says that the diameter of a shrinking gradient Ricci-harmonic soliton metric on a compact manifold satisfies

$$
\operatorname{diam}_{M} \geq \frac{2(\sqrt{2}-1) \pi}{\sqrt{\lambda}}
$$

By Theorem 3.5 we know that a nontrivial $\tau$-quasi Ricci-harmonic metric on a compact manifold $M$ should satisfy $\lambda>0$ and $\mu>0$. By a similar discussion as in [39], we can derive the following gap estimate for the diameter of a nontrivial $\tau$-quasi Ricci-harmonic metric on a compact manifold.

Theorem 6.5. Let g be a $\tau$-quasi Ricci-harmonic metric on a compact manifold $M$. Then the diameter of $M$ satisfies

$$
\frac{1}{\sqrt{-\tau_{0} \tau \lambda-\tau_{0}^{2} \tau^{2} \lambda e^{-\frac{2}{\tau} \omega_{f}}}}\left(\frac{\pi}{2}-\arcsin e^{\tau_{0} \omega_{f}}\right) \leq \operatorname{diam}_{M} \leq \pi \sqrt{\frac{m+\tau-1}{\lambda}}
$$

where

$$
\tau_{0}=\max \left\{-1,-\frac{1}{\tau}\right\}
$$

and

$$
\omega_{f}=\max _{x \in M} f(x)-\min _{x \in M} f(x) .
$$

In particular, if $\tau \geq 1$, then

$$
\frac{\pi}{2 \sqrt{\lambda}} \leq \operatorname{diam}_{M} \leq \pi \sqrt{\frac{m+\tau-1}{\lambda}} .
$$

Proof. Since

$$
\operatorname{Ric}_{f, \tau}=\lambda \mathrm{g}+\alpha \nabla \phi \otimes \nabla \phi \geq \lambda \mathrm{g}>0 .
$$

The upper bound in (6.6) comes from the Myers theorem in the weighted case [31]. For the lower bound in (6.6), we replace Ric and $R$ by $\operatorname{Ric}_{\phi}$ and $R_{\phi}$ respectively in [39], due to (1.6), (2.4), (2.5) and the fact that

$$
\text { Ric }=\operatorname{Ric}_{\phi}+\alpha \nabla \phi \otimes \nabla \phi \geq \text { Ric }_{\phi},
$$

by almost the same discussion as in [39], we can get the lower bound in (6.6).

Acknowledgements. The author would like to thank the referee for valuable comments and suggestions.

\section{References}

[1] Besse, A. L.: Einstein manifolds. - Ergeb. Math. Grenzgeb. (3) 10, Springer-Verlag, Berlin, 1987.

[2] CAO, H. D.: Geometry of Ricci solitons. - Chinese Ann. Math. Ser. B 27:2, 2006, 121-142.

[3] CaO, H. D., and D. Zhou: On complete gradient shrinking Ricci solitons. - J. Diff. Geom. $85: 2,2010,175-186$. 
[4] CaO, H. D., and X. P. Zhu: A complete proof of the Poincaré and geometrization conjectures. Application of the Hamilton-Perelman theory of the Ricci flow. - Asian J. Math. 10, 2006, 165492.

[5] Case, J.: On the nonexistence of quasi-Einstein metrics. - Pacific J. Math. 248:2, 2010, 277284.

[6] Case, J., Y. J. Shu, and G. Wei: Rigidity of quasi-Einstein metrics. - Differential Geom. Appl. 29:1, 2011, 93-100.

[7] Catino, G., C. Mantegazza, L. Mazzieri, and M. Rimoldi: Locally conformally flat quasi-Einstein manifolds. - J. Reine Angew. Math. 675, 2013, 181-189.

[8] Chen, B. L.: Strong uniqueness of the Ricci flow. - J. Diff. Geom. 82, 2009, 363-382.

[9] Chow, B., and D. Knopf: The Ricci flow: An introduction. - Math. Surveys MKonogr. 110, Amer. Math. Soc.

[10] Eells, J., and J. Sampson: Harmonic mappings of Riemannian manifolds. - Amer. J. Math. 86, 1964, 109-169.

[11] Eminenti, M., G. L. Nave, and C. Mantegazza: Ricci solitons: the equation point of view. - Manuscripta Math. 127, 2008, 345-367.

[12] Enders, J., R. Müller, and P. Topping: On type I singularities in Ricci flow. - Comm. Anal. Geom. 19:5, 2011, 905-922.

[13] Gilbarg, D., and N. Trudinger: Elliptic partial differential equations of second order. Springer-Verlag, Berlin-Heidelberg-New York, 1977.

[14] Gu, H. L., and X. P. ZHu: The existence of type II singularities for the Ricci flow on $S^{n+1}$. Comm. Anal. Geom. 16:3, 2008, 467-494.

[15] KIm, D. S., and Y. H. KIM: Compact Einstein warped product spaces with nonpositive scalar curvature. - Proc. Amer. Math. Soc. 131, 2003, 2573-2576.

[16] LI, X. D.: Liouville theorems for symmetric diffusion operators on complete Riemannian manifolds. - J. Math. Pures Appl. 84:10, 2005, 1295-1361.

[17] LI, Y.: Eigenvalues and entropies under the harmonic-Ricci flow. - Pacific J. Math. 267, 2014, 141-184.

[18] List, B.: Evolution of an extended Ricci flow system. - Ph.D. thesis, AEI Potsdam, 2006, http://www.diss.fu-berlin.de/2006/180/index.html.

[19] List, B.: Evolution of an extended Ricci flow system. - Comm. Anal. Geom. 16, 2008, 10071048.

[20] LÜ, H., D. N. PAge, and C. N. Pope: New inhomogeneous Einstein metrics on sphere bundles over Einstein-Kähler manifolds. - Phys. Lett. B 593:1-4, 2004, 218-226.

[21] Mantegazza, C., and R. Müller: Perelman's entropy functional at type I singularities of the Ricci flow. - J. Reine Angew. Math. (to appear).

[22] Morgan, J., and G. Tian: Ricci flow and the Poincaré conjecture. - Clay Math. Monogr. 3, Amer. Math. Soc., 2007.

[23] Müller, R.: The Ricci flow coupled with harmonic map heat flow. - Ph.D. thesis, ETH Zürich, 2009, http://e-collection.library.ethz.ch//view/eth:41938.

[24] MÜller, R.: Ricci flow coupled with harmonic map flow. - Ann. Sci. Éc. Norm. Supér. 45:4, 2012, 101-142.

[25] Müller, R.: Monotone volume formulas for geometric flows. - J. Reine Angew. Math. 643, 2010, 39-57.

[26] Munteanu, O., and J. P. Wang: Smooth metric measure spaces with nonnegative curvature. - Comm. Anal. Geom. 19:3, 2011, 451-486.

[27] Munteanu, O., and J. P. Wang: Analysis of the weighted Laplacian and applications to Ricci solitons. - Comm. Anal. Geom. 20:1, 2012, 55-94. 
[28] NABER, A.: Noncompact shrinking four solitons with nonnegative curvature. - J. Reine Angew. Math. 645, 2010, 125-153.

[29] Nash, J.: The embedding problem for Riemannian manifolds. - Comm. Pure Appl. Math. 63:2, 1956, 20-63.

[30] Perelman, G.: The entropy formula for the Ricci flow and its geometric applications. arxiv:Math.DG/0211159.

[31] QIAN, Z. M.: Estimates for weight volumes and applications. - Q. J. Math. 48:2, 1997, 235-242.

[32] Rigola, S., S. Rigoli, and A. Setti: Maximum principles on Riemannian manifolds and applications. - Mem. Amer. Math. Soc. 174:822, 2005.

[33] Rigola, S., M. Rimoldi, and A. Setti: Remarks on noncompact gradient Ricci solitons. Math. Z. 268:3-4, 2011, 777-790.

[34] TADANo, H.: A note on lower diameter bounds for closed domain manifolds of shrinking Ricci-harmonic solitons. - arXiv:1406.2861v2.

[35] Wang, L. F.: The upper bound of the $L_{\mu}^{2}$ spectrum. - Ann. Global Anal. Geom. 37:4, 2010, 393-402.

[36] Wang, L. F.: Rigid properties of quasi-Einstein metrics. - Proc. Amer. Math. Soc. 139:10, 2011, 3679-3689.

[37] Wang, L. F.: On noncompact $\tau$-quasi-Einstein metrics. - Pacific J. Math. 254:2, 2011, 449464.

[38] Wang, L. F.: On $L_{f}^{p}$-spectrum and $\tau$-quasi-Einstein metric. - J. Math. Anal. Appl. 389, 2012, 195-204.

[39] WANG, L. F.: Diameter estimate for compact quasi-Einstein metrics. - Math. Z. 273:3-4, 2013, 801-809.

[40] Wang, L. F.: Potential function estimates for quasi-Einstein metrics. - J. Funct. Anal. 267, 2014, 1986-2004.

[41] Wei, G., and W. Wylie: Comparison geometry for the Bakry-Émery Ricci tensor. - J. Diff. Geom. 83:2, 2009, 377-405.

[42] Williams, M.: Results on coupled Ricci and harmonic map flows. - Adv. Geom. 15:1, 2015, $7-26$.

[43] Wu, J. Y.: Upper bounds on the first eigenvalue for a diffusion operator via Bakry-Émery Ricci curvature. - J. Math. Anal. Appl. 361, 2010, 10-18.

[44] YANG, F., and J. Shen: Volume growth for gradient shrinking solitons of Ricci-harmonic flow. - Sci. China Math. 55, 2012, 1221-1228.

[45] Zhang, Z. H.: On the completeness of gradient Ricci solitons. - Proc. Amer. Math. Soc. 137, $2009,2755-2759$.

Received 22 December $2014 \bullet$ Revised received 4 September $2015 \bullet$ Accepted 2 October 2015 